COMMUNICATIONS IN

ANALYSIS AND GEOMETRY

Volume 14, Number 3, 411-441, 2006

\title{
Some Multi-valued Solutions to Monge-Ampère Equations
}

\author{
L. Caffarelli ${ }^{1}$ and YanYan $\mathrm{LI}^{2}$
}

\section{Introduction.}

In this paper, we construct several types of multi-valued solutions to the Monge-Ampère equation in higher dimensions. Recently, there has been considerable interest in understanding the behavior of a metric generated by a solution of the Monge-Ampère equation at a singularity. See, for instance Loftin, Yau and Zaslow [14] and Leung [12]. To explain our results let us refer first to the theory of multi-valued harmonic functions. Typical two dimensional examples of multi-valued harmonic functions are

$$
\begin{aligned}
& f_{1}(z)=\operatorname{Re}\left(z^{\frac{1}{k}}\right), \\
& f_{2}(z)=\arg (z)
\end{aligned}
$$

and

$$
f_{3}(z)=\operatorname{Re}(\sqrt{(z-1)(z+1)}) .
$$

The first one, $f_{1}$, is finitely valued, since it repeats itself once you have gone $k$-times around the origin. In the case of $f_{2}$, each time we go around the origin, the function increases by $2 \pi . f_{3}$ is a 2 -sheet harmonic function defined as follows. Write $z-1=r e^{i \theta}, z+1=s e^{i \phi}, \sqrt{(z-1)(z+1)}=\sqrt{r s} e^{i \frac{\theta+\phi}{2}}$, and $f_{3}(z)=\sqrt{r s} \cos \frac{\theta+\phi}{2}$. Each time the point $z$ goes around -1 , or 1 , and cross the intervel $(-1,1)$, the value of $f_{3}(z)$ is changed by a multiplication of -1 . Therefore, $f_{3}$ is actually defined on the 2 -sheet cover of $\mathbb{C} \backslash\{-1,1\}$.

Multi-valued harmonic functions have been studied by G.V. Evans ([8], [9] and [10]), H. Lewy [13] and L. Caffarelli ([1] and [2]), which have inspired the present paper.

\footnotetext{
${ }^{1}$ Partially supported by NFS grant DMS-0140388 and G-37-X71-G4

${ }^{2}$ Partially supported by NSF grant DMS-0401118.
} 


\section{Finitely Valued Solutions of the Monge-Ampère Equation.}

The geometric situation is the following: Let $D \subset \mathbb{R}^{n}, n \geq 2$, be a bounded strictly convex open set with smooth boundary $\partial D$, and let $\Sigma \subset D$ be homeomorphic in $\mathbb{R}^{n}$ to a $(n-1)$-dimensional closed disc, i.e., there exists a homeomorphism $\psi: \mathbb{R}^{n} \rightarrow \mathbb{R}^{n}$ such that $\psi(\Sigma)$ is a $(n-1)$-dimensional closed disc. Let $\Gamma=\partial \Sigma$, the boundary of $\Sigma$. Thus, $\Gamma$ is homeomorphic to a $(n-2)$-dimensional sphere for $n \geq 3$. In $\mathbb{R}^{3}, \Gamma$ is a curve "spanned" by a disc.

Let

$$
M=(D \backslash \Gamma) \times \mathbb{Z},
$$

denote a covering of $D \backslash \Gamma$ with the following standard parameterization: Fixing an $x^{*} \in D \backslash \Sigma$, and connecting $x^{*}$ by a smooth curve in $D \backslash \Gamma$ to a point $x$ in $D \backslash \Gamma$. If the curve goes through $\Sigma m \geq 0$ times in the positive direction (fixing such a direction), then we arrive at $(x, m)$ in $M$. If the curve goes through $\Sigma m \geq 0$ times in the negative direction, then we arrive at $(x,-m)$ in $M$. For $n \geq 3$, the fundamental group of $D \backslash \Gamma$ is $\mathbb{Z}$ and $M$ is the universal cover of $D \backslash \Gamma$.

For $k=2,3,4, \cdots$, we introduce an equivalence relation " $\sim_{k}$ " on $M$ as follows: $(x, m)$ and $(y, l)$ in $M$ are " $\sim_{k}$ " equivalent if $x=y$ and $m-l$ is an integer multiple of $k$. We let

$$
M_{k}:=M / \sim_{k}
$$

denote the $k$-sheet cover of $D \backslash \Gamma$, and let

$$
\partial^{\prime} M_{k}:=\cup_{i=1}^{k}(\partial D \times\{i\}) .
$$

For $\varphi_{1}, \cdots, \varphi_{k} \in C^{0}(\partial D)$, it is easy to prove, by Perron's method, that there exists $h \in C^{\infty}\left(M_{k}\right) \cap L^{\infty}\left(M_{k}\right) \cap C^{0}\left(M_{k} \cup \partial^{\prime} M_{k}\right)$ satisfying

$$
\left\{\begin{aligned}
\Delta h & =0, & & \text { on } M_{k} \\
h & =\varphi_{i}, & & \text { on } \partial D \times\{i\}, 1 \leq i \leq k .
\end{aligned}\right.
$$

Since $\Gamma$ has zero capacity, the maximum principle holds on $M_{k}$ : Let $u, v \in$ $L^{\infty}\left(M_{k}\right)$ satisfy $\Delta u \geq 0 \geq \Delta v$ in $M_{k}$ and $\liminf {\operatorname{dist}\left(y, \partial^{\prime} M_{k}\right)}(u(y)-v(y)) \leq 0$, then $u \leq v$ in $M_{k}$.

Let $\bar{h} \in C^{\infty}(D) \cap C^{0}(\bar{D})$ be the solution to

$$
\left\{\begin{aligned}
\Delta \bar{h} & =0, & & \text { in } D, \\
\bar{h} & =\frac{1}{k} \sum_{i=1}^{k} \varphi_{i}, & & \text { on } \partial D .
\end{aligned}\right.
$$


It was proved by Caffarelli [2], under some mild additional regularity assumption on $\Gamma$ (e.g. $\Gamma$ is $C^{1}$ ), that

$$
\lim _{x \rightarrow \bar{x}} h(x, m)=\bar{h}(\bar{x}), \quad \forall \bar{x} \in \Gamma, 1 \leq m \leq k,
$$

and, for some $0<\alpha<1$ and $C>0$, that

$$
|h(x, m)-\bar{h}(\bar{x})| \leq C|x-\bar{x}|^{\alpha}, \quad \forall(x, m) \in M_{k} .
$$

It follows, by the maximum principle, that (2.1) has a unique bounded solution $h$.

Let $\varphi_{1}, \cdots, \varphi_{k} \in C^{0}(\partial D)$, and let $f \in C^{0}\left(M_{k}\right)$ satisfy, for some positive constants $a$ and $b$,

$$
a \leq f \leq b \text { on } M_{k} .
$$

We consider the following Monge-Ampère equation on $M_{k}$ with Dirichlet boundary condition:

$$
\left\{\begin{aligned}
\operatorname{det}\left(D^{2} u\right) & =f, & & \text { on } M_{k} \\
u & =\varphi_{i}, & & \text { on } \partial D \times\{i\}, 1 \leq i \leq k .
\end{aligned}\right.
$$

Theorem 2.1. Let $M_{k}$ be as above, $k=2,3,4, \cdots, \varphi_{1}, \cdots, \varphi_{k} \in C^{0}(\partial D)$, $h$ be the bounded solution of (2.1), and let $f \in C^{0}\left(M_{k}\right)$ satisfy (2.2) for some positive constants $a$ and $b$. Then (2.3) has at least one bounded locally convex viscosity solution $u$ satisfying $u \leq h$ on $M_{k}$.

Proof. of Theorem 2.1. Let $P(x)$ be a convex quadratic polynomial satisfying

$$
\begin{aligned}
\operatorname{det}\left(D^{2} P\right) \geq b & \text { on } \quad D, \\
P<\inf _{M_{k}} h & \text { on } \quad \bar{D},
\end{aligned}
$$

and let $D^{\prime}$ be an open set in $D$ containing $\Sigma$ and satisfying $\operatorname{dist}\left(D^{\prime}, \partial D\right)>0$. As in Caffarelli, Nirenberg and Spruck [7], we construct $\underline{u}_{i} \in C^{\infty}(D) \cap$ $C^{0}(\bar{D}), 1 \leq i \leq k$, which satisfy

$$
\begin{aligned}
& \operatorname{det}\left(\underline{u}_{i}\right) \geq b, \text { on } D \text {, } \\
& \underline{u}_{i}=\varphi_{i} \text {, on } \partial D \text {, } \\
& \underline{u}_{i}<P \text {, on } D^{\prime} \text {. }
\end{aligned}
$$

Define

$$
\underline{u}(x, m)=\max \left\{\underline{u}_{m}(x), P(x)\right\}, \quad x \in D \backslash \Gamma, 1 \leq m \leq k .
$$


Then, $\underline{u} \in C^{0}\left(M_{k} \cup \partial^{\prime} M_{k}\right)$ is a locally convex subsolution of (2.3) satisfying

$$
\underline{u}(x, m)=P(x) \quad \forall x \in D^{\prime}, 1 \leq m \leq k .
$$

Let $\mathcal{S}$ denote the set of locally convex functions $v$ in $C^{0}\left(M_{k} \cup \partial^{\prime} M_{k}\right)$ which are viscosity subsolutions to (2.3) satisfying

$$
\limsup _{x \rightarrow \bar{x}} \max _{1 \leq m \leq k}[v(x, m)-h(x, m)] \leq 0, \quad \forall \bar{x} \in \Gamma .
$$

Clearly, $\underline{u} \in \mathcal{S}$.

Define on $M_{k}$

$$
u(x, m)=\sup \{v(x, m) \mid v \in \mathcal{S}\}, \quad 1 \leq m \leq k .
$$

For every $v \in \mathcal{S}$,

$$
\Delta v \geq 0 \text { on } M_{k} .
$$

By the maximum principle, using (2.4), $v \leq h$ on $M_{k}$. Thus, $u \leq h$ on $M_{k}$, and $u \in C^{0}\left(M_{k} \cup \partial^{\prime} M_{k}\right)$ is a locally convex viscosity solution of (2.3). Theorem 2.1 is established.

Let $\mathcal{S}^{*}$ denote the set of locally convex functions $v$ in $C^{0}\left(M_{k} \cup \partial^{\prime} M_{k}\right)$ which are viscosity solutions to (2.3) satisfying (2.4). Then,

$$
u^{*}(x, m):=\sup \left\{v(x, m) \mid v \in \mathcal{S}^{*}\right\}, \quad x \in D \backslash \Gamma, 1 \leq m \leq k
$$

is the largest element in $\mathcal{S}^{*}$. Moreover, by the maximum principle,

$$
u^{*} \leq h \text { in } M_{k} \text {. }
$$

It is clear from the proof of Theorem 2.1 that (2.3) has infinitely many solutions. In the following, we study the existence of solutions to (2.3) with prescribed values on $\Gamma$.

Theorem 2.2. Let $M_{k}, \varphi_{i}, f$ and $h$ be as in Theorem 2.1, and let $\underline{u} \in$ $C^{0}\left(M_{k} \cup \partial^{\prime} M_{k}\right)$ be a locally convex viscosity subsolution of (2.3) satisfying

$$
\underline{u}(\bar{x}):=\lim _{x \rightarrow \bar{x}} \underline{u}(x, m) \text { exists, finite, independent of } 1 \leq m \leq k, \forall \bar{x} \in \Gamma,
$$

and

$$
\underline{u}(\bar{x}) \leq \liminf _{x \rightarrow \bar{x}} \min _{1 \leq m \leq k} h(x, m), \quad \forall \bar{x} \in \Gamma .
$$

Then, there exists an unique locally convex viscosity solution u of (2.3) satisfying

$$
u(\bar{x}):=\lim _{x \rightarrow \bar{x}} u(x, m)=\underline{u}(\bar{x}), \quad 1 \leq m \leq k, \forall \bar{x} \in \Gamma .
$$


Proof. of Theorem 2.2. Let $\mathcal{S}$ denote the set of locally convex viscosity subsolutions $v$ of $(2.3)$ in $C^{0}\left(M_{k} \cup \partial^{\prime} M_{k}\right)$ satisfying

$$
\limsup _{x \rightarrow \bar{x}} v(x, m) \leq \underline{u}(\bar{x}), \quad 1 \leq m \leq k, \forall \bar{x} \in \Gamma .
$$

Clearly, $\underline{u} \in \mathcal{S}$. Define on $M_{k}$,

$$
u(x, m):=\sup \{v(x, m) \mid v \in \mathcal{S}\}, \quad 1 \leq m \leq k, x \in \bar{D} \backslash \Gamma .
$$

By the maximum principle,

$$
\underline{u} \leq u \leq h \text { on } M_{k} .
$$

It follows that $u \in C^{0}\left(M_{k} \cup \partial^{\prime} M_{k}\right)$ is a locally convex viscosity solution of (2.3) satisfying

$$
\liminf _{x \rightarrow \bar{x}} u(x, m) \geq \underline{u}(\bar{x}), \quad \forall 1 \leq m \leq k, \forall \bar{x} \in \Gamma .
$$

Let $v \in \mathcal{S}, \bar{x} \in \Gamma$ and $x \in D \backslash \Gamma$. Since the Hausdorff measure $H^{n-1}(\Gamma)=0$, there exist $y_{i} \rightarrow \bar{x}$ and $\nu_{i} \rightarrow \frac{x-\bar{x}}{\|x-\bar{x}\|}$ such that

$$
\left\{y_{i}+t \nu_{i} \mid t \geq 0\right\} \cap \Gamma=\emptyset .
$$

By the convexity and the boundedness of $v$ on the lifting of the segment $\left\{y_{i}+t \nu_{i} \mid t \geq 0\right\} \cap D$, we have, for some constant $C$ independent of $i$,

$$
v\left(y_{i}+|x-\bar{x}| \nu_{i}, m\right) \leq \max _{1 \leq m^{\prime} \leq k} v\left(y_{i}, m^{\prime}\right)+C|x-\bar{x}|, \quad \forall 1 \leq m \leq k .
$$

Sending $i$ to infinity, we have, by (2.5),

$$
v(x, m) \leq \underline{u}(\bar{x})+C|x-\bar{x}|, \quad \forall x \in D \backslash \Gamma, \forall 1 \leq m \leq k .
$$

It follows that

$$
\limsup _{x \rightarrow \bar{x}} u(x, m) \leq \underline{u}(\bar{x}) .
$$

Theorem 2.2 is established.

Example: Let $M_{k}$ and $f$ be as in Theorem 2.1, and let $\varphi_{1}, \cdots, \varphi_{k} \in C^{0}(\partial D)$ satisfy

$$
\varphi_{i} \geq \varphi_{1} \text { on } \partial D, 1 \leq i \leq k .
$$


Let $G \in C^{0}(\bar{D})$ be a convex function satisfying, in the viscosity sense,

$$
\left\{\begin{aligned}
\operatorname{det}\left(D^{2} G\right) & \geq f & \text { in } D \\
G & =\varphi_{1} & \text { on } \partial D
\end{aligned}\right.
$$

Then, (2.3) has a unique locally convex viscosity solution with $G$ as the prescribed value on $\Gamma$.

Let $D^{\prime}$ be a convex open set, containing $\Gamma$ satisfying $\overline{D^{\prime}} \subset D$. We follow [7] to construct convex $\underline{u}_{2}^{\prime}, \cdots, \underline{u}_{k}^{\prime} \in C^{\infty}(D) \cap C^{0}(\bar{D})$ satisfying

$$
\begin{array}{rll}
\operatorname{det}\left(D^{2} \underline{u}_{i}^{\prime}\right) \geq f & \text { in } \quad D, 2 \leq i \leq k, \\
\underline{u}_{i}^{\prime}=\varphi_{i}, & \text { on } \quad \partial D, 2 \leq i \leq k, \\
G>\underline{u}_{i}^{\prime} & \text { in } \quad D^{\prime}, 2 \leq i \leq k .
\end{array}
$$

Let $\underline{u}_{1}^{\prime}=G$,

$$
\underline{u}_{i}(x):=\max \left\{\underline{u}_{1}^{\prime}(x), \underline{u}_{i}^{\prime}(x)\right\}, \quad x \in \bar{D},
$$

and

$$
\underline{u}(x, m):=\underline{u}_{m}(x), \quad x \in D \backslash \Gamma, 1 \leq m \leq k .
$$

Then, $\underline{u} \in C^{0}\left(M_{k} \cup \partial^{\prime} M_{k}\right)$ is a locally convex viscosity subsolution of (2.3) satisfying

$$
\underline{u}(x, m)=G(x), \quad \forall 1 \leq m \leq k, \text { for } x \text { in } D \backslash \Gamma \text { and close to } \Gamma .
$$

As a result, by Theorem 2.2, we can solve (2.3) with $G$ as the prescribed value on $\Gamma$.

\section{Classical Solutions When $\Gamma$ is a "Plane Curve".}

Solutions given by Theorem 2.1 and Theorem 2.2 are not necessarily classical solutions. In this section, we study the existence of classical solutions of (2.3) with value 0 on $\Gamma$ under some further hypothesis on $\Gamma$.

Let $\Omega \subset D$ be two bounded open strictly convex subsets with smooth boundaries, denoted respectively by $\partial \Omega$ and $\partial D$. Let $\Sigma$, diffeomorphic to a $(n-1)$-disc, be the intersection of $\Omega$ and a hyperplane in $\mathbb{R}^{n}$, and let $\Gamma$ be the boundary of $\partial \Sigma$. The fundamental group of $D \backslash \Gamma$ is $\pi_{1}(D \backslash \Gamma)=\mathbb{Z}$ when $n \geq 3$. Let $M=(D \backslash \Gamma) \times \mathbb{Z}$ and $M_{k}=M / \sim_{k}$ be covering spaces of $D \backslash \Gamma$ as in Section 1. $\Sigma$ divides $\Omega$ into two open parts, denoted as $\Omega^{+}$and $\Omega^{-}$. Fixing a $x^{*} \in \Omega^{-}$, we use the convention that going through $\Sigma$ from $\Omega^{-}$to $\Omega^{+}$denotes the positive direction through $\Sigma$. 
Theorem 3.1. Let $M_{k}, k=2,3,4, \cdots$, be as above and let $f \in C^{\infty}\left(M_{k}\right)$ satisfy (2.2) for some positive constants $a$ and $b$. Then, there exists some $\beta>0$ such that for any $\varphi_{1}, \cdots, \varphi_{k} \in C^{\infty}(\partial D)$ satisfying

$$
\varphi_{i}>\beta \quad \text { on } \partial D, 1 \leq i \leq k,
$$

there exists a unique locally convex $u \in C^{\infty}\left(M_{k} \cup \partial^{\prime} M_{k}\right)$ satisfying (2.3) and

$$
\lim _{x \rightarrow \bar{x}} u(x, m)=0, \quad \forall 1 \leq m \leq k, \forall \bar{x} \in \Gamma .
$$

Proof. of Theorem 3.1. Let $v \in C^{\infty}(\bar{\Omega})$ be a convex function satisfying

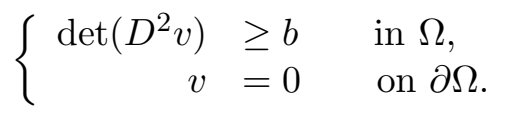

By Lemma 9.1, there exists $\bar{x}(\xi) \in \mathbb{R}^{n}$ for every $\xi \in \partial \Omega$ such that

$$
w_{\xi}(x):=\frac{1}{2}\left(|x-\bar{x}(\xi)|^{2}-|\xi-\bar{x}(\xi)|^{2}\right)<b^{-\frac{1}{n}} v(x), \quad \forall x \in \bar{\Omega} \backslash\{\xi\} .
$$

Moreover, $\sup _{\xi \in \partial \Omega}|\bar{x}(\xi)|<\infty$.

Set

$$
V(x)= \begin{cases}v(x), & x \in \bar{\Omega}, \\ \sup _{\xi \in \partial \Omega}\left(b^{\frac{1}{n}} w_{\xi}(x)\right), & x \in \bar{D} \backslash \bar{\Omega} .\end{cases}
$$

Then, $V \in C^{0}(\bar{D})$ is a convex viscosity subsolution to

$$
\operatorname{det}\left(D^{2} V\right)=b \text { in } D .
$$

Let

$$
\beta=\max _{\partial D} V
$$

With this value of $\beta$, and for any $\varphi_{1}, \cdots, \varphi_{k} \in C^{\infty}(\partial D)$ satisfying (3.1), we can construct as in [7] convex $\underline{u}_{1}^{\prime}, \cdots, \underline{u}_{k}^{\prime} \in C^{\infty}(D) \cap C^{0}(\bar{D})$ satisfying

$$
\begin{aligned}
\operatorname{det}\left(D^{2} \underline{u}_{i}^{\prime}\right) \geq b & \text { in } \quad D, 1 \leq i \leq k, \\
\underline{u}_{i}^{\prime}=\varphi_{i} & \text { on } \quad \partial D, 1 \leq i \leq k, \\
V>\underline{u}_{i}^{\prime} & \text { in } \quad \bar{\Omega}, 1 \leq i \leq k .
\end{aligned}
$$

Let

$$
\underline{u}_{i}(x)=\max \left\{V(x), \underline{u}_{i}^{\prime}(x)\right\}, \quad x \in D, 1 \leq i \leq k,
$$


and

$$
\underline{u}(x, m)=\underline{u}_{m}(x), \quad x \in D \backslash \Gamma, 1 \leq m \leq k .
$$

Clearly, $\underline{u}$ is a locally convex viscosity subsolution of $(2.3), \underline{u} \in C^{0}\left(M_{k} \cup\right.$ $\left.\partial^{\prime} M_{k}\right)$ and

$$
\underline{u}(x, m)=V(x), \quad \forall 1 \leq m \leq k, \text { for } x \text { in } D \backslash \Gamma \text { close to } \Gamma .
$$

In particular,

$$
\underline{u}(\bar{x})=\lim _{x \rightarrow \bar{x}} \underline{u}(x, m)=V(\bar{x})=0, \quad \forall 1 \leq m \leq k, \forall \bar{x} \in \Gamma .
$$

By Theorem 2.2, there exists a unique locally convex viscosity solution $u$ of (2.3) satisfying (3.2).

To complete the proof of Theorem 3.1, we need to show that $u \in$ $C^{\infty}\left(M_{k} \cup \partial^{\prime} M_{k}\right)$. This follows from the regularity theory developed by the first author in [3], [4] and [5]. Indeed if $u$ does not belong to $C^{\infty}\left(M_{k} \cup \partial^{\prime} M_{k}\right)$, then, by theorem 1 in [3] and theorem 2 in [4], there must be a line in $M_{k}$ on which $u$ is linear. By the arguments in the proof of corollary 4 in [3], the line cannot hit $\partial^{\prime} M_{k}$. So this singular line $\gamma(t), 0<t<1$, must be the lifting of $\left\{t x^{(1)}+(1-t) x^{(2)} \mid 0<t<1\right\}$ for some $x^{(1)}, x^{(2)} \in \Gamma, x^{(1)} \neq x^{(2)}$. By (3.2), $u(\gamma(t))=0$ for all $0<t<1$ and therefore, $u=0$ on the $(n-1)$ dimensional disc spanned by $\{\gamma(t)\}_{0<t<1}$ and $\Gamma$. This violates the theorem in [5]. Theorem 3.1 is established.

\section{Infinitely Valued Solutions with Exponentially Growing Right-Hand Side.}

This section is motivated by the following 2-d example: In the spirit of $\arg (z)$ that grows by a constant every time, we go around the origin, we construct a solution of the Monge-Ampère equation that grows by a factor every time, we go around the origin. In dimension 2 , we get the solution

$$
u(r, \theta)=r^{2} e^{\lambda \theta}
$$

that satisfies

$$
\operatorname{det}\left(D^{2} u\right)=2\left(2 \lambda^{2}-2 \lambda+1\right) e^{\lambda \theta} .
$$

We also mention some 3 -d examples which are in similar spirit. First, let $\Gamma$ be a regular embedded closed plane curve containing a region $\Sigma$ in the plane, and let $u$ denote the conductor potential of $\Sigma$ (see [11]). $u$ is the unique harmonic function in $\mathbb{R}^{3} \backslash \Sigma$ which takes the value 1 on $\Sigma$ and 0 at 
infinity. Reflecting $u$ by $2-u$ across $\Sigma$, we obtain a 2 -sheet harmonic function which takes value 0 at the infinity of one sheet and 2 for the other. Outside a compact set, the domain of $u$ is the disjoint union of two $\mathbb{R}^{3} \backslash\{$ a ball $\}$. Next, the solid angle (see [11] or [1]) gives an infinite-valued harmonic function: Let $\Gamma$ and $\Sigma$ be as above. For any point $x$ in $\mathbb{R}^{3}$, we generate by $\Sigma$ a cone with vertex at $x$, and we obtain an infinite-valued harmonic function by assigning the value $u(x)$ as the area of the intersection of the cone and the unit sphere centered at $x$. The function $u$ is infinite-valued since each time when $x$ penetrates $\Sigma$ from one side to the other, the intersection of the cone and the unit sphere changes from one half of the unit sphere to the other half with a minus sign and therefore, $u$ has to be extended across $\Sigma$ by adding $4 \pi$ (the area of the unit sphere).

We do now a similar construction for the Monge-Ampère equation. For $D, \Omega, \Gamma, M$ as in the last section, we study in this section multi-valued solutions to Monge-Ampère equations on $M$.

Let $S \in C^{\infty}(M)$ satisfy

$$
S(x, k)=S(x, k-1)+1, \quad \forall x \in D \backslash \Gamma, \forall k \in \mathbb{Z},
$$

and

$$
c:=\sup _{|k| \leq 2, x \in \bar{\Omega}} e^{S(x, k)}<\infty .
$$

We use notation

$$
\partial^{\prime} M=\cup_{i=-\infty}^{\infty}(\partial D \times\{i\}) .
$$

Theorem 4.1. Let $D, \Omega, \Gamma, M, S$ be as above. Then, there exists some positive constant $\beta$ such that for any $\varphi \in C^{\infty}(\partial D)$ satisfying

$$
\varphi>\beta \text { on } \partial D
$$

there exists a locally convex $u \in C^{\infty}\left(M \cup \partial^{\prime} M\right)$ satisfying

$$
\begin{gathered}
\operatorname{det}\left(D^{2} u\right)=e^{S}, \quad \text { in } \quad M, \\
u(x, k)=e^{\frac{1}{n}} u(x, k-1), \quad \forall(x, k) \in M, \\
\lim _{x \rightarrow \bar{x}} u(x, m)=0, \quad \forall \bar{x} \in \Gamma, \forall 1 \leq m \leq k, \\
u(x, k)=e^{\frac{k}{n}} \varphi(x), \quad \forall(x, k) \in \partial^{\prime} M .
\end{gathered}
$$


Proof. of Theorem 4.1. Let $\xi \in C^{\infty}(\bar{\Omega})$ be a convex function satisfying

$$
\left\{\begin{aligned}
\operatorname{det}\left(D^{2} \xi\right) & \geq c, \quad \text { in } \Omega \\
\xi & =0, \quad \text { on } \partial \Omega .
\end{aligned}\right.
$$

As in the proof of Theorem 3.1, we can extend $\xi$ to a convex $\widetilde{\xi} \in C^{0}(\bar{D})$ which satisfies in the viscosity sense

$$
\operatorname{det}\left(D^{2} \widetilde{\xi}\right) \geq c \text { in } D
$$

Let

$$
\beta:=\max _{\partial D} \widetilde{\xi}
$$

With this value of $\beta$, for any $\varphi \in C^{\infty}(\partial D)$ satisfying (4.1), we construct, as in [7], some convex $\eta^{\prime} \in C^{\infty}(D) \cap C^{0}(\bar{D})$ which satisfies

$$
\begin{array}{rll}
\operatorname{det}\left(D^{2} \eta^{\prime}\right) \geq c & \text { in } & D, \\
\eta^{\prime}=\varphi & \text { on } & \partial D, \\
\eta^{\prime}<\widetilde{\xi} & \text { on } \bar{\Omega} .
\end{array}
$$

Let

$$
\eta(x):=\max \left\{\eta^{\prime}(x), \widetilde{\xi}(x)\right\}, \quad x \in \bar{D} .
$$

Then, $\eta \in C^{0}(\bar{D})$ is a locally convex function satisfying

$$
\begin{aligned}
& \eta=\varphi \text { on } \partial D \\
& \eta=\widetilde{\xi} \text { in an open neighborhood of } \bar{\Omega}
\end{aligned}
$$

and, in the viscosity sense,

$$
\operatorname{det}\left(D^{2} \eta\right) \geq c \text { in } D .
$$

In particular,

$$
\begin{array}{lll}
\eta=\xi & \text { on } & \bar{\Omega}, \\
\eta=0 & \text { on } & \partial \Omega, \\
\eta<0 & \text { in } & \Omega .
\end{array}
$$

Define, for $k \in \mathbb{Z}$,

$$
\underline{u}(x, k)=\left\{\begin{aligned}
e^{\frac{k-1}{n}} \eta(x), & x \in \Omega^{+}, \\
e^{\frac{k}{n}} \eta(x), & x \in \bar{D} \backslash \overline{\Omega^{+}} .
\end{aligned}\right.
$$


It is not difficult to see that this extends to $\underline{u} \in C^{0}\left(M \cup \partial^{\prime} M\right)$ which is locally convex and satisfies

$$
\begin{aligned}
\underline{u}(x, k) & =e^{\frac{1}{n}} \underline{u}(x, k-1), \quad \forall(x, k) \in M, \\
\lim _{x \rightarrow \bar{x}} \underline{u}(x, m) & =0, \quad \forall \bar{x} \in \Gamma, \quad \forall 1 \leq m \leq k, \\
\underline{u}(x, k) & =e^{\frac{k}{n}} \varphi(x), \quad \forall(x, k) \in \partial D \times \mathbb{Z},
\end{aligned}
$$

and, in the viscosity sense,

$$
\operatorname{det}\left(D^{2} \underline{u}\right) \geq e^{S} \text { in } M .
$$

Let $\mathcal{S}$ denote the set of locally convex functions $v \in C^{0}\left(M \cup \partial^{\prime} M\right)$ satisfying

$$
\begin{aligned}
v(x, k) & =e^{\frac{1}{n}} v(x, k-1), \quad \forall(x, k) \in M, \\
\lim _{x \rightarrow \bar{x}} v(x, m) & =0, \quad \forall \bar{x} \in \Gamma, \quad \forall 1 \leq m \leq k, \\
v(x, k) & =e^{\frac{k}{n}} \varphi(x), \quad \forall(x, k) \in \partial^{\prime} M,
\end{aligned}
$$

and, in the viscosity sense,

$$
\operatorname{det}\left(D^{2} v\right) \geq e^{S} \text { in } M
$$

Let $B_{1}, B_{2}, B_{3}, \cdots$ be open balls in $D \backslash \Gamma$ such that

$$
D \backslash \Gamma=\cap_{i=1}^{\infty} \cup_{j=i}^{\infty} B_{j},
$$

i.e., every point in $D \backslash \Gamma$ belongs to infinitely many balls.

For any $v \in \mathcal{S}$, and for any open ball $B \subset D \backslash \Gamma$, we define $T_{B} v$ as follows: The lifting of $B$ into $M$ is the union of infinite disjoint balls, denoted as $\left\{B^{(m)}\right\}_{m=-\infty}^{\infty}$. We keep $T_{B} v$ the same as $v$ outside $\cup_{m=-\infty}^{\infty} B^{(m)}$, while in each $B^{(m)}$, we replace $v$ by the solution of

$$
\left\{\begin{aligned}
\operatorname{det}\left(D^{2}\left(T_{B} v\right)\right) & =e^{S}, \quad \text { in } B^{(m)}, \\
\left(T_{B} v\right) & =v, \quad \text { on } \partial B^{(m)} .
\end{aligned}\right.
$$

It is not difficult to see that $T_{B} v \in \mathcal{S}$, and $T_{B} v \geq v$ in $M$. Let $B_{1}^{\prime}, B_{2}^{\prime}, B_{3}^{\prime}, B_{4}^{\prime}, \cdots$ be a sequence of balls defined by $B_{1}^{\prime}=B_{1}, B_{2}^{\prime}=B_{2}$, $B_{3}^{\prime}=B_{1}, B_{4}^{\prime}=B_{2}, B_{5}^{\prime}=B_{3}, B_{6}^{\prime}=B_{1}, B_{7}^{\prime}=B_{2}, B_{8}^{\prime}=B_{3}, B_{9}^{\prime}=B_{4}$, $B_{10}^{\prime}=B_{1}, \cdots$, and let $v_{0}=\underline{u}$ and $v_{i}=T_{B_{i}^{\prime}} v_{i-1}$ for $i=1,2,3, \cdots$. Thus, we have defined a sequence of functions $\left\{v_{i}\right\}$ in $\mathcal{S}$ which satisfy

$$
v_{0} \leq v_{1} \leq v_{2} \leq v_{3} \leq \cdots \text { in } M .
$$


For $x \in \partial D$, let $\nu(x)$, denote the unit inner normal of $\partial D$ at $x$. We will show below that there exist some positive constants $\epsilon$ and $C$ such that

$$
\begin{aligned}
v_{i}(x+t \nu(x), k) & \leq v_{i}(x, k)+C e^{\frac{k}{n}} t \\
& =e^{\frac{k}{n}}+C e^{\frac{k+2}{n}} t, \quad \forall x \in \partial D, k \in \mathbb{Z}, 0<t<\epsilon,
\end{aligned}
$$

and

$$
v_{i}(x, k) \leq C e^{\frac{k}{n}}, \quad \forall x \in D \backslash \Gamma, \forall k \in \mathbb{Z} .
$$

Since $\Gamma$ is closed, there exists $\epsilon>0$ such that $\operatorname{dist}(\Gamma, \partial D)>\epsilon$. For any $x \in \partial D$, since $H^{n-1}(\Gamma)=0$, there exists $\left|\nu_{l}\right|=1, \nu_{l} \rightarrow \nu(x)$, such that

$$
\left\{x+t \nu_{l} \mid t>0\right\} \cap \Gamma=\emptyset .
$$

Let $t_{l}>\epsilon, x+t_{l} \nu_{l} \in \partial D$ and let $\gamma(t), 0 \leq t \leq t_{l}, \gamma(0)=(x, k)$, be the lifting of $\left\{x+t \nu_{l} \mid 0 \leq t \leq t_{l}\right\}$ to $M$, then $v_{i}(\gamma(t))$ is a convex function for $t \in\left[0, t_{l}\right]$. Since $\Gamma \in \partial \Omega$ and $\Omega$ is strictly convex, the segment $\left\{x+t \nu_{l} \mid 0 \leq t \leq t_{l}\right\}$ can intersect $\Sigma$ at most once. Therefore, for $\left|k^{\prime}-k\right| \leq 1$,

$$
\begin{aligned}
v_{i}\left(x+t \nu_{l}, k\right) & \leq v_{i}(x, k)+\left(\frac{v_{i}\left(x+t_{l} \nu_{l}, k^{\prime}\right)-v_{i}(x, k)}{t_{l}}\right) t \\
& =v_{i}(x, k)+\left(\frac{e^{\frac{k^{\prime}}{n}} \varphi\left(x+t \nu_{l}\right)-e^{\frac{k}{n}} \varphi(x)}{t_{l}}\right) t \leq v_{i}(x, k)+C e^{\frac{k}{n}} t .
\end{aligned}
$$

Estimate (4.7) is established.

For $x \in D$, dist $(x, \partial D)<\epsilon$, and $k \in \mathbb{Z}$, we deduce from (4.7) that

$$
v_{i}(x, k) \leq C e^{\frac{k}{n}} .
$$

Since $H^{n-1}(\Gamma)=0$, for any $x \in D \backslash \Gamma$ with $\operatorname{dist}(x, \partial D)>\epsilon$, there exists $|\nu|=1$ such that

$$
\{x+t \nu \mid t \in \mathbb{R}\} \cap \Gamma=\emptyset .
$$

Let $t^{-}<0<t^{+}$satisfy $x+t^{ \pm} \nu \in \partial D$. Let $\gamma(t), t^{-} \leq t \leq t^{+}, \gamma(0)=(x, k)$, be the lifting of $\left\{x+t \nu \mid t^{-} \leq t \leq t^{+}\right\}$into $M$. As before, $v_{i}\left(\gamma\left(t^{ \pm}\right)\right)$ are bounded from above by $C e^{\frac{k}{n}}$. Thus, by the convexity of $v_{i}(\gamma(t))$ in $t$, $v_{i}(x)$ is bounded from above by $C e^{\frac{k}{n}}$. Estimate (4.8) is established. With (4.7) and (4.8), and some standard arguments, $v_{i}$ monotonically converge to some locally convex $u \in C^{0}\left(M \cup \partial^{\prime} M\right)$ which satisfy (4.3), (4.5), and, in the viscosity sense, (4.2). Using some arguments similar to those in the proof of Theorem 2.2, we see that $u$ satisfies (4.4). The smoothness of $u$ follows from the regularity theory of the first author as used in the proof of Theorem 3.1. Theorem 4.1 is established. 


\section{Global Finitely Valued Solutions.}

We present here existence results closely related to theorem 1.7 in [6]. Let $\Omega$, $\Gamma, \Sigma$ be as at the beginning of Section 2 , and we take $D$ to be $\mathbb{R}^{n}$ instead of a bounded strictly convex open set. We restrict to $n \geq 3$. For $k=2,3, \cdots$, we define $M$ and $M_{k}$ as at the beginning of Section 2 with $D$ replaced by $\mathbb{R}^{n}$.

Let

$\mathcal{A}=\{A \mid A$ is real $n \times n$ symmetric positive definite matrix with $\operatorname{det}(A)=1\}$.

For $f \in C^{0}\left(M_{k}\right)$ satisfying, for some positive constants $a$ and $b$,

$$
a \leq \inf _{M_{k}} f \leq \sup _{M_{k}} f \leq b,
$$

and

$$
\{f \neq 1\} \text { is compact, }
$$

we consider

$$
\operatorname{det}\left(D^{2} u\right)=f \text { on } M_{k}
$$

Theorem 5.1. For $n \geq 3, k \geq 2$, let $M_{k}, \Gamma$ be as above, and let $f \in C^{0}\left(M_{k}\right)$ satisfy (5.1) and (5.2) for some positive constants $a$ and $b$. Then, for any $c_{m} \in \mathbb{R}, b_{m} \in \mathbb{R}^{n}$ and $A_{m} \in \mathcal{A}, 1 \leq m \leq k$, there exists some $\beta_{*} \in \mathbb{R}$ such that for any $\beta>\beta_{*}$ there exists a unique locally convex viscosity solution $u \in C^{0}\left(M_{k}\right)$ of (5.3) which satisfy

$$
\begin{gathered}
\limsup _{|x| \rightarrow \infty}\left(|x|^{n-2}\left|u(x, m)-\left[\frac{1}{2} x^{\prime} A_{m} x+b_{m} \cdot x+c_{m}\right]\right|\right)<\infty, \quad \forall 1 \leq m \leq k \\
\lim _{x \rightarrow \bar{x}} u(x, m)=-\beta, \quad \forall \bar{x} \in \Gamma, \forall 1 \leq m \leq k
\end{gathered}
$$

Moreover, $u \in C^{\infty}\left(M_{k}\right)$ provided that $f \in C^{\infty}\left(M_{k}\right)$.

Proof. of Theorem 5.1. For simplicity, we assume that $f \equiv 1$. The general case can be obtained by incorporating some arguments in [6]. Let $\Phi \in$ $C^{\infty}(\bar{\Omega})$ be a convex function satisfying

$$
\begin{cases}\operatorname{det}\left(D^{2} \Phi\right)>1 & \text { on } \bar{\Omega}, \\ \Phi=0, & \text { on } \partial \Omega\end{cases}
$$


By Lemma 9.1, there exists $\bar{x}(\xi) \in \mathbb{R}^{n}$ for every $\xi \in \partial \Omega$ such that

$$
w_{\xi}(x):=\frac{1}{2}\left(|x-\bar{x}(\xi)|^{2}-|\xi-\bar{x}(\xi)|^{2}\right)<\Phi(x), \quad \forall x \in \bar{\Omega} \backslash\{\xi\} .
$$

Moreover, $\sup _{\xi \in \partial \Omega}|\bar{x}(\xi)|<\infty$.

Define,

$$
V(x)= \begin{cases}\Phi(x), & x \in \bar{\Omega}, \\ \sup _{\xi \in \partial \Omega} w_{\xi}(x), & x \in \mathbb{R}^{n} \backslash \bar{\Omega} .\end{cases}
$$

Then, $V$ is a convex function satisfying, in the viscosity sense,

$$
\operatorname{det}\left(D^{2} V\right) \geq 1 \quad \text { in } \mathbb{R}^{n}
$$

Fix some $R_{1}>0$ such that

$$
\Omega \subset B_{R_{1}}
$$

Write

$$
\frac{1}{2} x^{\prime} A_{m} x+b_{m} \cdot x+c_{m}=\frac{1}{2}\left|\left(A_{m}\right)^{\frac{1}{2}} x+\left(A_{m}\right)^{-\frac{1}{2}} b\right|^{2}+c-\frac{1}{2}\left|\left(A_{m}\right)^{-\frac{1}{2}} b\right|^{2} .
$$

Let

$$
R_{2}:=2 \max _{1 \leq m \leq k} \max _{|x| \leq R_{1}}\left|\left(A_{m}\right)^{\frac{1}{2}} x+\left(A_{m}\right)^{-\frac{1}{2}} b\right| .
$$

Define, for $a>1$,

$$
\begin{aligned}
& w_{m, a}(x):=\inf _{B_{R_{2}}} V+\int_{2 R_{2}}^{\left|\left(A_{m}\right)^{\frac{1}{2}} x+\left(A_{m}\right)^{-\frac{1}{2}} b\right|}\left(s^{n}+a\right)^{\frac{1}{n}} d s, \quad 0<|x|<\infty . \\
& w_{m, a} \text { satisfies } \\
& \qquad \operatorname{det}\left(D^{2} w_{m, a}(x)\right)=1 \quad \forall 0<|x|<\infty .
\end{aligned}
$$

By the definition of $R_{2}$,

$$
\begin{aligned}
w_{m, a}(x) & \leq \inf _{B_{R_{2}}} V+\int_{2 R_{2}}^{R_{2} / 2}\left(s^{n}+a\right)^{\frac{1}{n}} d s \\
& <\inf _{B_{R_{2}}} V \leq V(x), \quad \forall 1 \leq m \leq k, \forall|x| \leq R_{1} .
\end{aligned}
$$

Fixing some $R_{3}>3 R_{2}$ satisfying

$$
\min _{1 \leq m \leq k} \min _{|x|=R_{3}}\left|\left(A_{m}\right)^{\frac{1}{2}} x+\left(A_{m}\right)^{-\frac{1}{2}} b\right|>3 R_{2},
$$


we choose $a_{1}>1$ such that

$w_{m, a}(x)>\inf _{B_{R_{2}}} V+\int_{2 R_{2}}^{3 R_{2}}\left(s^{n}+a\right)^{\frac{1}{n}} d s>V(x), \forall|x|=R_{3}, \forall 1 \leq m \leq k, \forall a \geq a_{1}$.

It is easy to see, in view of (5.6), that

$$
w_{m, a}(x)=\frac{1}{2} x^{\prime} A_{m} x+b_{m} \cdot x+c_{m}+\mu(m, a)+O\left(|x|^{2-n}\right) \quad \text { as }|x| \rightarrow \infty,
$$

where $\mu(m, a)$, monotonic and continuous in $a$ for large $a$, tends to $\infty$ as $a \rightarrow \infty$.

Define, for $a \geq a_{1}$ and $1 \leq m \leq k$,

$$
\underline{u}_{m, a}(x)= \begin{cases}\max \left\{V(x), w_{m, a}(x)\right\}-\mu(m, a), & |x| \leq R_{3} \\ w_{m, a}(x)-\mu(m, a), & |x| \geq R_{3}\end{cases}
$$

Then, for $1 \leq m \leq k$,

$$
\begin{gathered}
\underline{u}_{m, a}(x)=\frac{1}{2} x^{\prime} A_{m} x+b_{m} \cdot x+c_{m}+O\left(|x|^{2-n}\right) \quad \text { as }|x| \rightarrow \infty, \\
\underline{u}_{m, a}=-\mu(m, a) \quad \text { on } \Gamma, \\
\underline{u}_{m, a}=V \quad \text { in some open neighborhood of } \bar{\Sigma},
\end{gathered}
$$

and $\underline{u}_{m, a}$ is a convex function satisfying, in the viscosity sense,

$$
\operatorname{det}\left(D^{2} \underline{u}_{m, a}\right) \geq 1 \quad \text { in } \mathbb{R}^{n} \text {. }
$$

It is easy to see that there exist continuous functions $a^{(m)}(a), 2 \leq m \leq k$, satisfying

$$
\lim _{a \rightarrow \infty} a^{(m)}(a)=\infty
$$

and, for $2 \leq m \leq k$,

$$
\mu\left(m, a^{(m)}(a)\right)=\mu(1, a) \text { for large } a .
$$

Define, with the convention $a^{(1)}(a)=a$,

$$
\underline{u}_{a}(x, m)=\underline{u}_{m, a^{(m)}(a)}(x), \quad \forall(x, m) \in M_{k} .
$$

Then, $\underline{u}_{a}$ is a locally convex function on $M_{k}$ satisfying

$$
\underline{u}_{a}(x, m)=\frac{1}{2} x^{\prime} A_{m} x+b_{m} \cdot x+c_{m}+O\left(|x|^{2-n}\right) \quad \text { as }|x| \rightarrow \infty,
$$




$$
\lim _{x \rightarrow \bar{x}} \underline{u}_{a}(x, m)=\mu(1, a), \quad \forall \bar{x} \in \Gamma, \forall 1 \leq m \leq k,
$$

and, in the viscosity sense,

$$
\operatorname{det}\left(D^{2} \underline{u}_{a}\right) \geq 1 \quad \text { in } M_{k} .
$$

Next, we produce appropriate supersolutions. Let $R_{4}$ be defined by

$$
\max _{1 \leq m \leq k} \max _{|x|=2 R_{3}}\left|\left(A_{m}\right)^{\frac{1}{2}} x+\left(A_{m}\right)^{-\frac{1}{2}} b\right|=R_{4}
$$

and let

$$
w_{m}^{+}(x)=\left\{\begin{array}{lr}
\int_{R_{4}}^{\left|\left(A_{m}\right)^{\frac{1}{2}} x+\left(A_{m}\right)^{-\frac{1}{2}} b\right|}\left[s^{n}-\left(R_{4}\right)^{n}\right]^{\frac{1}{n}} d s, & \left|\left(A_{m}\right)^{\frac{1}{2}} x+\left(A_{m}\right)^{-\frac{1}{2}} b\right| \geq R_{4}, \\
0, & \left|\left(A_{m}\right)^{\frac{1}{2}} x+\left(A_{m}\right)^{-\frac{1}{2}} b\right|<R_{4} .
\end{array}\right.
$$

Then, $w_{m}^{+} \in C^{1}\left(\mathbb{R}^{n}\right) \cap C^{\infty}\left(\mathbb{R}^{n} \backslash B_{R_{4}}\right)$ is a convex function, satisfying

$$
\begin{gathered}
\operatorname{det}\left(D^{2} w_{m}^{+}\right)(x)=1 \quad \text { for }\left|\left(A_{m}\right)^{\frac{1}{2}} x+\left(A_{m}\right)^{-\frac{1}{2}} b\right|>R_{4}, \\
\nabla w_{m}^{+}(x)=0 \quad \text { for }\left|\left(A_{m}\right)^{\frac{1}{2}} x+\left(A_{m}\right)^{-\frac{1}{2}} b\right|=R_{4}, \\
w_{m}^{+}(x)=0 \quad \forall|x|<\frac{3}{2} R_{3},
\end{gathered}
$$

and, for some $\bar{\beta}(m) \in \mathbb{R}$,

$$
w_{m}^{+}(x)=\frac{1}{2} x^{\prime} A_{m} x+b_{m} \cdot x+c_{m}+\bar{\beta}(m)+O\left(|x|^{2-n}\right), \quad \text { as }|x| \rightarrow \infty .
$$

Define,

$$
w^{+}(x, m):=w_{m}^{+}(x)-\bar{\beta}(m), \quad \forall(x, m) \in M_{k} .
$$

Clearly, $w^{+}$satisfies, in the viscosity sense

$$
\left.\operatorname{det}\left(D^{2} w^{+}\right)\right) \leq 1, \quad \text { on } M_{k} .
$$

For $\lambda$ large, $w^{+}+\lambda>\underline{u}_{a}$ on $M_{k}$. Let

$$
\bar{\lambda}_{a}:=\inf \left\{\lambda>0 \mid w^{+}+\lambda>\underline{u}_{a} \text { on } M_{k}\right\} .
$$

Fix some $a_{2} \geq a_{1}$, such that

$$
-\mu(1, a)<-\max _{1 \leq m \leq k} \bar{\beta}(m) \quad \forall a \geq a_{2} .
$$


By (5.7), (5.8), (5.9) and (5.10), no touching of $w^{+}+\bar{\lambda}_{a}$ and $\underline{u}_{a}$ can occur (see arguments on page 575 of [6]). Thus $\bar{\lambda}_{a}=0$ and $w^{+}>\underline{u}_{a}$ on $M_{k}$ for all $a \geq a_{2}$.

Let $\mathcal{S}_{a}$ denote the set of locally convex functions $v$ on $M_{k}$ satisfying

$$
\begin{aligned}
& v \leq w^{+} \quad \text { on } \quad M_{k}, \\
& \operatorname{det}\left(D^{2} v\right) \geq 1 \quad \text { on } \quad M_{k}, \\
& \limsup _{x \rightarrow \bar{x}} v(x, m) \leq-\mu(1, a), \quad \forall \bar{x} \in \Gamma, \forall 1 \leq m \leq k .
\end{aligned}
$$

Clearly, $\underline{u}_{a} \in \mathcal{S}_{a}$. Define

$$
u_{a}(x, m)=\sup \{v(x, m) \mid v \in \mathcal{S}\}, \quad \forall(x, m) \in M_{k} .
$$

Using some arguments similar to those in the proof of Theorem 2.2, together with some standard arguments, we see that $u_{a}$, for $a \geq a_{2}$, is a locally convex solution to (5.3) with $f \equiv 1$ satisfying (5.4) and (5.5) with $\beta=\mu(1, a)$. To complete the proof of Theorem 5.1, we only need to prove that $u \in C^{\infty}\left(M_{k}\right)$. This follows from the regularity theory of the first author as used in the proof of Theorem 3.1. Indeed, the only additional observation is that, because of (5.4), there can not be a ray to infinity on which $u_{a}$ is linear. Theorem 5.1 is established.

\section{Infinitely Valued Solutions with a Triple Point.}

In this section, only in $\mathbb{R}^{3}$, we construct more complex multi-valued solutions. Here, the curve defining the multiple leaved space is like a "Mercedes Benz star" and each time, we cross one of the three holes, we go into a different copy of $\mathbb{R}^{3} \backslash \Gamma$. In particular, the origin is a triple point where the "three cuts" coexist. We point out that this construction is possible due to the particular geometry of the Pogorelov singular solution.

Let $\mathbb{R}^{3}=\left\{\left(x_{1}, x_{2}, x_{3}\right) \mid x_{i} \in \mathbb{R}\right\}$, and let $e_{1}, e_{2}, e_{3}$ be distinct unit vectors lying in the $\left(x_{1}, x_{2}\right)$-plane. We assume that

$$
e_{1} \cdot e_{2}>-1, \quad e_{2} \cdot e_{3}>-1, \quad e_{3} \cdot e_{1}>-1 .
$$

Let,

$$
e_{1}^{\prime}=\frac{e_{1}+e_{3}}{1+e_{1} \cdot e_{3}}, \quad e_{2}^{\prime}=\frac{e_{2}+e_{1}}{1+e_{2} \cdot e_{1}}, \quad e_{3}^{\prime}=\frac{e_{3}+e_{2}}{1+e_{3} \cdot e_{2}}
$$

and

$$
\ell_{1}^{\prime}(x):=e_{i}^{\prime} \cdot x, \quad i=1,2,3 .
$$


Clearly,

$$
\left\{\begin{array}{l}
\ell_{1}^{\prime}\left(e_{1}\right)=\ell_{1}^{\prime}\left(e_{3}\right)=1 \\
\ell_{2}^{\prime}\left(e_{2}\right)=\ell_{2}^{\prime}\left(e_{1}\right)=1 \\
\ell_{3}^{\prime}\left(e_{3}\right)=\ell_{3}^{\prime}\left(e_{2}\right)=1 .
\end{array}\right.
$$

Let $D$ be a strictly convex bounded open set containing the origin with diameter $\operatorname{diam}(D)=2$. Recall that the singular solution to Monge-Ampère equation of Pogorelov in 3-dimension:

$$
P\left(x_{1}, x_{2}, x_{3}\right)=f\left(x_{1}\right)\left|\left(x_{2}, x_{3}\right)\right|^{\frac{4}{3}}
$$

where $f$ is positive and smooth in, say, $(-2,2)$, and blows up at $x_{1}= \pm 2$. See, e.g., [5] for the ODE satisfied by $f$. The function $P$ satisfies in the viscosity sense

$$
\operatorname{det}\left(D^{2} P\right)=1
$$

Moreover, $P\left(x_{1}, 0,0\right)=0$ for all $\left|x_{1}\right|<2$.

Let $P_{e_{j}}(x)$ denotes the Pogorelov solution which vanishes along the $e_{j}$-line, and let

$$
h^{0}(x)=\max _{j}\left\{x \cdot e_{j}+P_{e_{j}}(x)\right\}, \quad x \in \bar{D} .
$$

Let,

$$
\beta^{\prime}:=\sup _{D}\left\{h^{0}, \ell_{1}^{\prime}, \ell_{2}^{\prime}, \ell_{3}^{\prime}\right\}>0 .
$$

For any $\varphi^{\prime} \in C^{0}(\partial D)$, satisfying $\min _{\partial D} \varphi^{\prime}>\beta^{\prime}$, we can construct as before convex $h^{\prime} \in C^{\infty}(D) \cap C^{0}(\bar{D})$ satisfying

$$
\begin{aligned}
\operatorname{det}\left(D^{2} h^{\prime}\right)>1 & \text { in } \quad D, \\
h^{\prime}=\varphi^{\prime} & \text { on } \quad \partial D, \\
h^{\prime}<0 & \text { in } \quad B_{1} .
\end{aligned}
$$

Define,

$$
h(x)=\max \left\{h^{0}(x), h^{\prime}(x)\right\}, \quad x \in \bar{D} .
$$

By (6.2), and the fact that $P_{e_{j}}=0$ along the $e_{j}$-line, we have, $\forall 0<s<1$,

$$
\begin{array}{ll}
\ell_{1}^{\prime}\left(s e_{1}\right)=h\left(s e_{1}\right)=s, & \ell_{1}^{\prime}\left(s e_{3}\right)=h\left(s e_{3}\right)=s, \\
\ell_{2}^{\prime}\left(s e_{2}\right)=h\left(s e_{2}\right)=s, & \ell_{2}^{\prime}\left(s e_{1}\right)=h\left(s e_{1}\right)=s, \\
\ell_{3}^{\prime}\left(s e_{3}\right)=h\left(s e_{3}\right)=s, & \ell_{3}^{\prime}\left(s e_{2}\right)=h\left(s e_{2}\right)=s .
\end{array}
$$


We, now consider the convex domain

$$
C_{j}:=\left\{x \in D \mid h(x)<\ell_{j}^{\prime}(x)\right\}
$$

and let

$$
\begin{gathered}
\Sigma_{j}=C_{j} \cap\left\{x \mid x_{3}=0\right\}, \\
\Gamma_{j}:=\partial C_{j} \cap\left\{x \mid x_{3}=0\right\}, \\
\Gamma=\Gamma_{1} \cup \Gamma_{2} \cup \Gamma_{3}, \\
\Omega_{j}^{+}=C_{j} \cap\left\{x \mid x_{3}>0\right\}, \quad \Omega_{j}^{-}=C_{j} \cap\left\{x \mid x_{3}<0\right\} .
\end{gathered}
$$

Let $M$ denote the universal cover of $D \backslash \Gamma$. The fundamental group $G$ of $M$ is the free products of three cyclic groups $G_{1}, G_{2}$ and $G_{3}$. We use $g_{i}$ to denote the generator of $G_{i}$. Fixing a base point $x^{*}$ in $D \backslash \Gamma$, we parameterize points of $M=(D \backslash \Gamma) \times G$ as usual: For $x \in D \backslash \Gamma$ and $g=g_{1}^{i_{1}} g_{2}^{i_{2}} g_{3}^{i_{3}} \cdots g_{1}^{i_{3 l+1}} g_{2}^{i_{3 l+2}} g_{3}^{i_{3 l+3}} \in G$, we use $(x, g)$ to denote the point of $M$ obtained by a path starting from $x^{*}$, ending at $x$, and crossing $\Sigma_{1} i_{1}$ times $\left(i_{1}=0\right.$ means no crossing, $i_{1}>0$ means crossing in the positive direction, i.e., from $\Omega_{1}^{-}$to $\Omega_{1}^{+}, i_{1}<0$ means crossing in the negative direction), crossing $\Sigma_{2} i_{2}$ times, crossing $\Sigma_{3} i_{3}$ times, crossing $\Sigma_{1} i_{4}$ times, crossing $\Sigma_{2} i_{5}$ times, $\cdots$, crossing $\Sigma_{3} i_{3 l+3}$ times. We use notation $\partial^{\prime} M=\{(x, g) \mid x \in \partial D$, $g \in G\}$.

Let $a_{1}, a_{2}, a_{3} \in \mathbb{R}$ and let $S$ be a smooth function defined on $M$ satisfying

$$
\begin{aligned}
S(x, g)= & S(x, \bar{g})+\left(i_{1}+i_{4}+\cdots+i_{3 l+1}\right) a_{1} \\
& +\left(i_{2}+i_{5}+\cdots+i_{3 l+2}\right) a_{2}+\left(i_{3}+i_{6}+\cdots+i_{3 l+3}\right) a_{3},
\end{aligned}
$$

where, $\bar{g}$ denotes the identity element of $G$ and

$$
g=g_{1}^{i_{1}} g_{2}^{i_{2}} g_{3}^{i_{3}} \cdots g_{1}^{i_{3 l+1}} g_{2}^{i_{3 l+2}} g_{3}^{i_{3 l+3}} \in G .
$$

We will produce in the rest of this section locally convex viscosity solutions $u$ to

$$
\operatorname{det}\left(D^{2} u\right)=e^{S} \text { in } M
$$

satisfying

$$
D^{2}(u(x, g)-\gamma(g) u(x, \bar{g}))=0, \quad \forall(x, g) \in M,
$$

where

$$
\begin{array}{r}
\gamma(g)=\gamma_{1}(g) \gamma_{2}(g) \gamma_{3}(g), \gamma_{1}(g)=e^{\frac{a_{1}}{n}\left(i_{1}+i_{4}+\cdots+i_{3 l+1}\right)}, \\
\gamma_{2}(g)=e^{\frac{a_{2}}{n}\left(i_{2}+i_{5}+\cdots+i_{3 l+2}\right)}, \gamma_{3}(g)=e^{\frac{a_{3}}{n}\left(i_{3}+i_{6}+\cdots+i_{3 l+3}\right)} .
\end{array}
$$


Let $b=e^{10\left(\left|a_{1}\right|+\left|a_{2}\right|+\left|a_{3}\right|\right)}, h^{*}=b h$, and $\beta=b \beta^{\prime}$. We consider $\varphi \in C^{0}(\partial D)$ satisfying

$$
\min _{\partial D} \varphi>\beta .
$$

We will first construct $\underline{u}$ on $M$ satisfying

$$
\begin{gathered}
\operatorname{det}\left(D^{2} \underline{u}\right) \geq e^{S} \quad \text { on } M, \\
\underline{u}(x, g)=\gamma(g) \underline{u}(x, \bar{g})-\gamma_{1}(g) \ell_{1}-\gamma_{2}(g) \ell_{2}-\gamma_{3}(g) \ell_{3}, \quad(x, g) \in M,
\end{gathered}
$$

and

$$
\underline{u}(x, g)=\gamma(g) \varphi-\gamma_{1}(g) \ell_{1}-\gamma_{2}(g) \ell_{2}-\gamma_{3}(g) \ell_{3}, \quad(x, g) \in \partial^{\prime} M,
$$

where $\ell_{j}=b \ell_{j}^{\prime}$.

We will use $\partial C_{j} \cap\left\{x \mid x_{3}>0\right\}$ as a cut-off surface, playing a similar role as $\partial \Omega \cap \partial \Omega^{+}$in the proof of Theorem 4.1. We change $h^{*}-\ell_{j}\left(\ell_{j}=b \ell_{j}^{\prime}\right)$ to $e^{\frac{a_{j}}{n}}\left(h^{*}-\ell_{j}\right)$ when crossing $\Sigma_{j}$ in the positive direction into $\Omega_{j}^{+}$, so on that leaf, we replace $h^{*}$ by $e^{\frac{a_{j}}{n}}\left(h^{*}-\ell_{j}\right)+\ell_{j}$. In general, for $e^{\frac{c}{n}} h^{*}+\ell(\ell$ is some linear function), we change it to $e^{\frac{c+a_{j}}{n}}\left(h^{*}-\ell_{j}\right)+e^{\frac{c}{n}} \ell_{j}+\ell$.

Following the above procedure we have defined $\underline{u}$ :

$$
\underline{u}(x, \bar{g})=\left\{\begin{array}{rl}
h^{*}(x) & x \in \bar{D} \backslash\left(\Omega_{1}^{+} \cup \Omega_{2}^{+} \cup \Omega_{3}^{+}\right), \\
e^{\frac{a_{j}}{n}}\left(h^{*}-\ell_{j}\right)+\ell_{j} & x \in \Omega_{j}^{+}, j=1,2,3,
\end{array}\right.
$$

and $\underline{u}$ satisfies (6.7), (6.8) and (6.9).

Theorem 6.1. For $\varphi \in C^{0}(\partial D)$ satisfying (6.6), there exists a unique locally convex viscosity solution $u \in C^{0}\left(M \cup \partial^{\prime} M\right)$ to (6.4) satisfying

$$
\begin{gathered}
u(x, g)=\gamma(g) u(x, \bar{g})-\gamma_{1}(g) \ell_{1}-\gamma_{2}(g) \ell_{2}-\gamma_{3}(g) \ell_{3}, \quad(x, g) \in M, \\
u(x, g)=\underline{u}(x, g), \quad \forall(x, g) \in \partial^{\prime} M,
\end{gathered}
$$

and

$$
\lim _{x \rightarrow \bar{x}}(u(x, g)-\underline{u}(x, g))=0, \quad \forall \bar{x} \in \Gamma, g \in G .
$$

Consequently u satisfies (6.5).

Remark 6.2. The above theorem can easily be extended to $m \geq 3$ unit vectors $e_{1}, \cdots, e_{m}$ lying in the $\left(x_{1}, x_{2}\right)$-plane satisfying

$$
e_{1} \cdot e_{2}>-1, e_{2} \cdot e_{3}>-1, \cdots, e_{m-1} \cdot e_{m}>-1, e_{m} \cdot e_{1}>-1 \text {. }
$$

In fact, $\left\{e_{1}, \cdots, e_{m}\right\}$ do not need to lie exactly in the $\left(x_{1}, x_{2}\right)$-plane. These can be seen from the proof of Theorem 6.1. 
Proof. of Theorem 6.1. Let $\mathcal{S}$ denote the set of locally convex functions $v \in C^{0}\left(M \cup \partial^{\prime} M\right)$ satisfying, in the viscosity sense,

$$
\begin{gathered}
\operatorname{det}\left(D^{2} v\right) \geq e^{S} \quad \text { in } M, \\
\limsup _{x \rightarrow \bar{x}}(v(x, g)-\underline{u}(x, g)) \leq 0, \quad \forall \bar{x} \in \Gamma, g \in G, \\
v(x, g)=\gamma(g) v(x, \bar{g})-\gamma_{1}(g) \ell_{1}-\gamma_{2}(g) \ell_{2}-\gamma_{3}(g) \ell_{3}, \quad(x, g) \in M,
\end{gathered}
$$

and

$$
v(x, g)=\gamma(g) \varphi-\gamma_{1}(g) \ell_{1}-\gamma_{2}(g) \ell_{2}-\gamma_{3}(g) \ell_{3}, \quad(x, g) \in \partial^{\prime} M .
$$

Clearly, $\underline{u} \in \mathcal{S}$. Define,

$$
u(x, g)=\sup \{v(x, g) \mid v \in \mathcal{S}\}, \quad(x, g) \in M .
$$

Modifying the arguments in the proof of Theorem 4.1, we see that $u$ belongs to $\mathcal{S}$ and satisfies (6.4) and (6.10). The uniqueness of such $u$ follows from standard arguments. Theorem 6.1 is established.

\section{Infinitely Valued Solutions with Constant Right-Hand Side.}

In this section, we construct infinitely valued solutions with constant righthand side. The invariance here, is given by the fact that $u$ in consecutive leaves differs from the previous one in an affine transformation.

For $n \geq 3$, we use $\mathbb{R}^{n-1}=\left\{x=\left(x_{1}, \cdots, x_{n-1}, 0\right) \mid x_{i} \in \mathbb{R}\right\}$ to denote the hyperplane in $\mathbb{R}^{n}=\left\{x=\left(x_{1}, \cdots, x_{n}\right) \mid x_{i} \in \mathbb{R}\right\}$. Let $\Sigma \subset \mathbb{R}^{n-1}$ be a $(n-1)$-dimensional strictly convex bounded open set with smooth boundary, and $T$ be a $n \times n$ real matrix satisfying $T x=x$ for all $x \in \mathbb{R}^{n-1}$, i.e.

$$
T=\left(\begin{array}{cccccc}
1 & 0 & 0 & \cdots & 0 & \lambda_{1} \\
0 & 1 & 0 & \cdots & 0 & \lambda_{2} \\
\cdots & \cdots & \cdots & \cdots & \cdots & \cdots \\
0 & 0 & 0 & \cdots & 1 & \lambda_{n-1} \\
0 & 0 & 0 & \cdots & 0 & 1
\end{array}\right)
$$

Let $\Gamma$ be the boundary of $\Sigma$ in the $\mathbb{R}^{n-1}$, and let

$$
M=\left(\mathbb{R}^{n} \backslash \Gamma\right) \times \mathbb{Z}
$$


be the universal cover of $\mathbb{R}^{n} \backslash \Gamma$ with the usual parameterization: Fixing a point $x^{*}$ in $\mathbb{R}^{n} \backslash \Gamma$, and connecting $x^{*}$ by a smooth curve in $\mathbb{R}^{n} \backslash \Gamma$ to a point $x$ in $\mathbb{R}^{n} \backslash \Gamma$. If the curve goes through $\Sigma m \geq 0$ times in the positive direction (say, increasing $x_{3}$ ), then we arrive at $(x, m)$ in $M$. If the curve goes through $\Sigma m \geq 0$ times in the negative direction, then we arrive at $(x,-m)$ in $M$.

For $k=2,3,4, \cdots$, we introduce an equivalence relation " $\sim_{k}$ " on $M$ as follows: $(x, m)$ and $(y, l)$ in $M$ are " $\sim_{k}$ " equivalent if $x=y$ and $m-l$ is an integer multiple of $k$. We let

$$
M_{k}:=M / \sim_{k}
$$

denote the $k$-sheet cover of $\mathbb{R}^{n} \backslash \Gamma$.

Theorem 7.1. Let $M$ and $T$ be as above, $b \in \mathbb{R}^{n}, c \in \mathbb{R}$, and let $A$ be a real symmetric $n \times n$ matrix with $\operatorname{det}(A)=1$. Then, there exists some constant $\beta_{*}>0$, which depends only on $T, \Gamma, A, b$ and $c$, such that for any $\beta \geq \beta_{*}$, there exists a unique locally convex function $u \in C^{\infty}(M)$ satisfying

$$
\begin{gathered}
\operatorname{det}\left(D^{2} u\right)=1 \quad \text { in } M, \\
u(x, m)=u(T x, m-1) \quad \forall(x, m) \in M, \\
\lim _{x \rightarrow \bar{x}} u(x, m)=-\beta, \quad \forall \bar{x} \in \Gamma, \forall m \in \mathcal{Z},
\end{gathered}
$$

and

$$
\limsup _{|x| \rightarrow \infty}\left(|x|^{n-2}\left|u(x, m)-Q\left(T^{m} x\right)\right|\right)<\infty, \quad \forall m \in \mathcal{Z},
$$

where $Q(x):=\frac{1}{2} x^{\prime} A x+b \cdot x+c$.

Remark 7.2. It is clear that the conclusion of Theorem 7.1 holds with $M$ replaced by $M_{k}, k=2,3,4, \cdots$.

Proof. of Theorem 7.1. By the affine invariance of the equation, we may assume without loss of generality that $b=0, c=0$ and $A$ is the identity matrix. Let $\Omega \subset \mathbb{R}^{n}$ be a strictly convex bounded open set with smooth boundary satisfying $\Sigma=\Omega \cap \mathbb{R}^{n-1}$ and, therefore, $\Gamma=\partial \Omega \cap \mathbb{R}^{n-1}$. Let $\Phi \in C^{\infty}(\bar{\Omega})$ be a convex function satisfying

$$
\begin{cases}\operatorname{det}\left(D^{2} \Phi\right)>1 & \text { on } \bar{\Omega} \\ \Phi=0, & \text { on } \partial \Omega\end{cases}
$$


and let

$$
\widetilde{\Phi}(x):=\Phi(x)+K\left|x_{n}\right|, \quad x \in \bar{\Omega},
$$

where $K>1$ is some fixed large constant, depending only on $T$ and $\Gamma$, satisfying, for all $\left(x_{1}, \cdots, x_{n-1}, 0\right) \in \bar{\Sigma}$, that

$$
\left\{\begin{array}{l}
\liminf _{s \rightarrow 0} \frac{\widetilde{\Phi}\left(x_{1}, \cdots, x_{n-1}, s\right)-\widetilde{\Phi}\left(x_{1}, \cdots, x_{n-1}, 0\right)}{|s|}>0, \\
\liminf _{s \rightarrow 0} \frac{\widetilde{\Phi}\left(T\left(x_{1}, \cdots, x_{n-1}, s\right)\right)-\widetilde{\Phi}\left(T\left(x_{1}, \cdots, x_{n-1}, 0\right)\right)}{|s|}>0,
\end{array}\right.
$$

Because of (7.5), $\widetilde{\Phi}$ also satisfies, in the viscosity sense,

$$
\operatorname{det}\left(D^{2} \widetilde{\Phi}\right)>1 \quad \text { in } \Omega .
$$

By Lemma 9.1, there exists $\bar{x}(\xi) \in \mathbb{R}^{n}$ for every $\xi \in \partial \Omega$ such that

$$
w_{\xi}(x):=\widetilde{\Phi}(\xi)+\frac{1}{2}\left(|x-\bar{x}(\xi)|^{2}-|\xi-\bar{x}(\xi)|^{2}\right)<\widetilde{\Phi}(x), \quad \forall x \in \bar{\Omega} \backslash\{\xi\} .
$$

Moreover, $\sup _{\xi \in \partial \Omega}|\bar{x}(\xi)|<\infty$.

Define,

$$
V(x)= \begin{cases}\widetilde{\Phi}(x), & x \in \bar{\Omega}, \\ \sup _{\xi \in \partial \Omega} w_{\xi}(x), & x \in \mathbb{R}^{n} \backslash \bar{\Omega} .\end{cases}
$$

Then $V$ is a convex function satisfying, in the viscosity sense,

$$
\operatorname{det}\left(D^{2} V\right) \geq 1 \quad \text { in } \mathbb{R}^{n} .
$$

Let $R_{1}>0$ satisfy,

$$
\Omega \subset B_{R_{1}},
$$

and let

$$
R_{2}=2 \max _{|m| \leq 4} \max _{|x| \leq R_{1}}\left|T^{m} x\right| .
$$

We consider, for $a>1$,

$$
w_{a}(x):=\inf _{B_{R_{2}}} V+\int_{2 R_{2}}^{|x|}\left(s^{n}+a\right)^{\frac{1}{n}} d s, \quad 0<|x|<\infty .
$$

By the definition of $R_{2}$,

$$
\begin{array}{r}
w_{a}\left(T^{m} x\right) \leq \inf _{B_{R_{2}}} V+\int_{2 R_{2}}^{R_{2} / 2}\left(s^{n}+a\right)^{\frac{1}{n}} d s<\inf _{B_{R_{2}}} V \leq V(x), \\
\forall|m| \leq 4, \forall|x| \leq R_{1} .
\end{array}
$$


Fixing some $R_{3}>3 R_{2}$ satisfying

$$
\min _{|m| \leq 4} \min _{|x|=R_{3}}\left|T^{m} x\right|>3 R_{2}
$$

we choose some $a_{1}>1$, such that

$$
w_{a}\left(T^{m} x\right)>\inf _{B_{R_{2}}} V+\int_{2 R_{2}}^{3 R_{2}}\left(s^{n}+a\right)^{\frac{1}{n}} d s>V(x), \quad \forall|x|=R_{3}, \quad \forall|m| \leq 4 .
$$

It is easy to see that

$$
w_{a}(x)=\frac{1}{2}|x|^{2}+\mu(a)+O\left(|x|^{2-n}\right) \quad \text { as }|x| \rightarrow \infty,
$$

where $\mu(a)$, monotonic and continuous in $a$ for large $a$, tends to $\infty$ as $a \rightarrow \infty$.

Define, for $a \geq a_{1}$,

$$
\underline{u}_{a}^{(0)}(x)= \begin{cases}\max \left\{V(x), w_{a}(x)\right\}-\mu(a), & |x| \leq R_{3} \\ w_{a}(x)-\mu(a), & |x| \geq R_{3}\end{cases}
$$

and

$$
\underline{u}_{a}^{(m)}(x)=\underline{u}_{a}^{(0)}\left(T^{m} x\right), \quad x \in \mathbb{R}^{n} \backslash \Sigma, m \in \mathcal{Z} .
$$

Then, for $m \in \mathcal{Z}$,

$$
\begin{gathered}
\underline{u}_{a}^{(m)}(x)=\frac{1}{2}\left|T^{m} x\right|^{2}+O\left(|x|^{2-n}\right) \quad \text { as }|x| \rightarrow \infty, \\
\underline{u}_{a}^{(m)}=-\mu(a) \quad \text { on } \Gamma, \\
\underline{u}_{a}^{(m)}=V \quad \text { in some open neighborhood of } \bar{\Sigma},
\end{gathered}
$$

and $\underline{u}_{a}^{(m)}$ is a convex function satisfying, in the viscosity sense,

$$
\operatorname{det}\left(D^{2} \underline{u}_{a}^{(m)}\right) \geq 1 \quad \text { in } \mathbb{R}^{n}
$$

Define,

$$
\underline{u}_{a}(x, m)=\underline{u}_{a}^{(m)}(x), \quad \forall(x, m) \in M .
$$

Then, $\underline{u}_{a}$ is a locally convex function on $M$ satisfying

$$
\begin{gathered}
\underline{u}_{a}(x, m)=\frac{1}{2}\left|T^{m} x\right|^{2}+O\left(|x|^{2-n}\right), \quad \text { as }|x| \rightarrow \infty, \\
\lim _{x \rightarrow \bar{x}} \underline{u}_{a}(x, m)=-\mu(a), \quad \forall \bar{x} \in \Gamma, \quad \forall m \in \mathcal{Z},
\end{gathered}
$$


and, in view of (7.5), in the viscosity sense,

$$
\operatorname{det}\left(D^{2} \underline{u}_{a}\right) \geq 1 \quad \text { in } M .
$$

Let $R_{4}$ be defined by

$$
\max _{|m| \leq 4} \max _{|x|=2 R_{3}}\left|T^{m} x\right|=R_{4}
$$

and let

$$
w(x)= \begin{cases}\int_{R_{4}}^{|x|}\left[s^{n}-\left(R_{4}\right)^{n}\right]^{\frac{1}{n}} d s, & |x| \geq R_{4}, \\ 0, & |x|<R_{4} .\end{cases}
$$

Then, $w \in C^{1}\left(\mathbb{R}^{n}\right) \cap C^{\infty}\left(\mathbb{R}^{n} \backslash B_{R_{4}}\right)$ is a convex function satisfying

$$
\begin{gathered}
\operatorname{det}\left(D^{2} w\right)=1 \quad \text { on } \mathbb{R}^{n} \backslash B_{R_{4}}, \\
\nabla w=0 \quad \text { on } \partial B_{R_{4}},
\end{gathered}
$$

and, for some $\beta \in \mathbb{R}$,

$$
w(x)=\frac{1}{2}|x|^{2}+\beta+O\left(|x|^{2-n}\right), \quad \text { as }|x| \rightarrow \infty .
$$

Define,

$$
\bar{w}(x, m)=w\left(T^{m} x\right)-\beta, \quad \forall(x, m) \in M .
$$

Clearly, $\bar{w}$ satisfies, in the viscosity sense

$$
\operatorname{det}\left(D^{2} \bar{w}\right) \leq 1, \quad \text { on } M
$$

For $\lambda$ large, $\bar{w}+\lambda>w_{a}$ on $M$. Let

$$
\bar{\lambda}_{a}:=\inf \left\{\lambda>0 \mid \bar{w}+\lambda>w_{a} \text { on } M\right\} .
$$

Fix some $a_{2} \geq a_{1}$ such that

$$
-\mu(a)<-\beta \quad \forall a \geq a_{2} .
$$

By (7.6), (7.7), (7.8) and (7.9), no touching of $\bar{w}+\bar{\lambda}_{a}$ and $w_{a}$ can occur (see arguments on page 575 of [6]). Thus $\bar{\lambda}_{a}=0$ and $\bar{w}>w_{a}$ on $M$ for all $a \geq a_{2}$.

Let $\mathcal{S}_{a}$ denote the set of locally convex functions $v$ on $M$ satisfying

$$
v \leq \bar{w} \quad \text { on } M
$$




$$
\begin{array}{cc}
v(x, m)=v(T x, m-1) & \forall(x, m) \in M, \\
\operatorname{det}\left(D^{2} v\right) \geq 1 & \text { on } M, \\
\limsup _{x \rightarrow \bar{x}} v(x, m) \leq-\mu(a), & \forall \bar{x} \in \Gamma, \forall m \in \mathcal{Z} .
\end{array}
$$

Clearly, $\underline{u}_{a} \in \mathcal{S}_{a}$. Define

$$
u_{a}(x, m)=\sup \{v(x, m) \mid v \in \mathcal{S}\}, \quad \forall(x, m) \in M .
$$

Using some arguments similar to those in the proofs of Theorem 2.2 and Theorem 4.1, together with some standard arguments, we see that $u_{a}$, for $a \geq a_{2}$, is a locally convex solution to (7.1) satisfying (7.4) with $Q(x) \equiv \frac{1}{2}|x|^{2}$ and (7.3) with $\beta=\mu(a)$. To complete the proof of Theorem 7.1, we only need to prove that $u \in C^{\infty}(M)$. This follows from the regularity theory of the first author as used in the proof of Theorem 3.1. Indeed, the only additional observation is that, because of (7.4), there can not be a ray to infinity on which $u_{a}$ is linear. Theorem 7.1 is established.

\section{Infinitely Valued Solutions With Constant Right-Hand Side and a Triple Point.}

In this section we construct, only in $\mathbb{R}^{3}$, infinitely valued solutions with constant right-hand side and a triple point by combining the arguments in Section 5 and 6. As in Section 5, the construction relies on the geometry of the Pogorelov singular solution.

In $\mathbb{R}^{3}=\left\{\left(x_{1}, x_{2}, x_{3}\right) \mid x_{i} \in \mathbb{R}\right\}$, let $e_{1}, e_{2}, e_{3}$ be distinct unit vectors lying in $\mathbb{R}^{2}:=\left\{\left(x_{1}, x_{2}, 0\right) \mid x_{i} \in \mathbb{R}\right\}$ which satisfy (6.1). With the Pogorelov singular solution in (6.3), we let $P_{e_{j}}$ denote the Pogorelov solution which vanishes along the $e_{j}$-line, and we define

$$
h(x)=\max _{j}\left\{x \cdot e_{j}+P_{e_{j}}(x)\right\}, \quad|x| \leq \frac{3}{2} .
$$

We let

$$
\widetilde{h}(x):=h(x)+K\left|x_{3}\right|, \quad|x| \leq \frac{3}{2},
$$

and we will fix some large constant $K$ below.

Let $T_{1}, T_{2}, T_{3}$ be $3 \times 3$ real matrices satisfying $T_{i} x=x$ for all $x \in \mathbb{R}^{2}$. We, now fix some large positive constant $K$ such that for all $\left|\left(x_{1}, x_{2}, 0\right)\right| \leq \frac{3}{2}$, for $i=1,2,3$, and for all $m=0, \pm 1, \pm 2$, we have

$$
\liminf _{s \rightarrow 0} \frac{\widetilde{h}\left(T_{i}^{m}\left(x_{1}, x_{2}, s\right)\right)-\widetilde{h}\left(T_{i}^{m}\left(x_{1}, x_{2}, 0\right)\right)}{|s|}>0,
$$


Let $b \in \mathbb{R}^{n}, A$ be a real symmetric $3 \times 3$ matrix, then there exists some $c_{*}$, which depends on $A, b$ and $\tilde{h}$, such that for all $c>c_{*}$, we can construct, as in Section 6, a convex function $W$ on $\mathbb{R}^{n}$, satisfying,

$$
\begin{gathered}
W(x)=\widetilde{h}(x), \quad \forall|x| \leq \frac{5}{4}, \\
W(x)=\frac{1}{2} x^{\prime} A x+b \cdot x+c+O\left(\frac{1}{|x|}\right), \quad \text { as }|x| \rightarrow \infty,
\end{gathered}
$$

and, in the viscosity sense,

$$
\operatorname{det}\left(D^{2} W\right) \geq 1 \quad \text { in } \mathbb{R}^{n} .
$$

Let $\Gamma_{1}, \Gamma_{2}, \Gamma_{3}$ be three bounded convex curves lying in $\mathbb{R}^{2}$ satisfying

$$
\begin{aligned}
& \Gamma_{1} \cap\left\{x \in \mathbb{R}^{2}|| x \mid<1\right\}=\left\{s e_{1} \mid 0 \leq s<1\right\} \cup\left\{s e_{2} \mid 0 \leq s<1\right\}, \\
& \Gamma_{2} \cap\left\{x \in \mathbb{R}^{2}|| x \mid<1\right\}=\left\{s e_{2} \mid 0 \leq s<1\right\} \cup\left\{s e_{3} \mid 0 \leq s<1\right\},
\end{aligned}
$$

and

$$
\Gamma_{3} \cap\left\{x \in \mathbb{R}^{2}|| x \mid<1\right\}=\left\{s e_{3} \mid 0 \leq s<1\right\} \cup\left\{s e_{1} \mid 0 \leq s<1\right\} .
$$

We set

$$
\Gamma=\Gamma_{1} \cup \Gamma_{2} \cup \Gamma_{3} .
$$

Let $M$ denote the universal cover of $\mathbb{R}^{3} \backslash \Gamma$. The fundamental group $G$ of $M$ is the free products of three cyclic groups $G_{1}, G_{2}$ and $G_{3}$. We use $g_{i}$ to denote the generator of $G_{i}$. Fixing a base point $x^{*}$ in $\mathbb{R}^{3} \backslash \Gamma$, we parameterize points of $M=\left(\mathbb{R}^{3} \backslash \Gamma\right) \times G$ as usual: For $x \in \mathbb{R}^{3} \backslash \Gamma$ and $g=g_{1}^{i_{1}} g_{2}^{i_{2}} g_{3}^{i_{3}} \cdots g_{1}^{i_{3 l+1}} g_{2}^{i_{3 l+2}} g_{3}^{i_{3 l+3}} \in G$, we use $(x, g)$ to denote the point of $M$ obtained by a path starting from $x^{*}$, ending at $x$, and crossing $\Sigma_{1} i_{1}$ times $\left(i_{1}=0\right.$ means no crossing, $i_{1}>0$ means crossing in the positive direction, i.e., from $\Omega_{1}^{-}$to $\Omega_{1}^{+}, i_{1}<0$ means crossing in the negative direction), crossing $\Sigma_{2} i_{2}$ times, crossing $\Sigma_{3} i_{3}$ times, crossing $\Sigma_{1} i_{4}$ times, crossing $\Sigma_{2} i_{5}$ times, $\cdots$, crossing $\Sigma_{3} i_{3 l+3}$ times.

For $g=g_{1}^{i_{1}} g_{2}^{i_{2}} g_{3}^{i_{3}} \cdots g_{1}^{i_{3 l+1}} g_{2}^{i_{3 l+2}} g_{3}^{i_{3 l+3}} \in G$, we use notation

$$
T(g)=T_{3}^{i_{3 l+3}} T_{2}^{i_{3 l+2}} T_{1}^{i_{3 l+1}} \cdots T_{3}^{i_{3}} T_{2}^{i_{2}} T_{1}^{i_{1}} .
$$

Now, we define a function $\underline{u}$ on $M$ by setting, for all $(x, g) \in M$,

$$
\underline{u}(x, g)=W(T(g) x) .
$$


It is clear that $\underline{u}$ is a locally convex function on $M$ satisfying

$$
\begin{gathered}
\underline{u}(x, \bar{g}) \equiv W(x), \\
\underline{u}(x, g) \equiv \underline{u}(T(g) x, \bar{g}),
\end{gathered}
$$

and, in the viscosity sense,

$$
\operatorname{det}\left(D^{2} \underline{u}\right) \geq 1 \quad \text { in } M .
$$

Clearly, $\underline{u}$ satisfies

$$
\limsup _{|x| \rightarrow \infty}(|x||\underline{u}(x)-Q(T(g) x)|)<\infty, \quad \forall g \in G,
$$

where $Q(x):=\frac{1}{2} x^{\prime} A x+b \cdot x+c$.

Modifying the construction of the super solution $\bar{w}$ in Section 6, and increasing the value of $c_{*}$ if necessary (recall that $c>c_{*}$ ), we may construct a locally convex function $\bar{u}$ on $M$ satisfying

$$
\begin{gathered}
\bar{u} \geq \underline{u} \quad \text { on } M, \\
\limsup _{|x| \rightarrow \infty}(|x||\bar{u}(x)-Q(T(g) x)|)<\infty, \quad \forall g \in G, \\
\bar{u}(x, g) \equiv \bar{u}(T(g) x, \bar{g}) \quad \forall(x, g) \in M,
\end{gathered}
$$

and, in the viscosity sense,

$$
\operatorname{det}\left(D^{2} \bar{u}\right) \leq 1 \quad \text { on } M .
$$

Let $\mathcal{S}$ denote the set of locally convex functions $v$ on $M$ satisfying,

$$
\begin{gathered}
v \leq \bar{u} \quad \text { in } M, \\
v(x, g)=v(T(g) x, \bar{g}) \quad \forall(x, g) \in M, \\
\limsup _{x \rightarrow \bar{x}}(v(x, g)-\underline{u}(x, g)) \leq 0 \quad \forall \bar{x} \in \Gamma, \forall g \in G,
\end{gathered}
$$

and, in the viscosity sense,

$$
\operatorname{det}\left(D^{2} v\right) \geq 1 \quad \text { in } M .
$$

Clearly, $\underline{u} \in \mathcal{S}$. Define

$$
u(x, g)=\sup \{v(x, g) \mid v \in \mathcal{S}\}, \quad \forall(x, g) \in M .
$$


Theorem 8.1. The above defined $u$ is a locally convex viscosity solution to

$$
\operatorname{det}\left(D^{2} u\right)=1 \quad \text { in } M
$$

satisfying

$$
\begin{gathered}
u(x, g)=u(T(g) x, \bar{g}) \quad \forall(x, g) \in M, \\
\lim _{x \rightarrow \bar{x}}(u(x, g)-\underline{u}(x, g))=0 \quad \forall \bar{x} \in \Gamma, \forall g \in G,
\end{gathered}
$$

and

$$
\limsup _{|x| \rightarrow \infty}(|x||u(x)-Q(T(g) x)|)<\infty, \quad \forall g \in G
$$

Proof. of Theorem 8.1. This theorem follows from some arguments similar to those used in the proofs of the theorems in previous sections. Let us outline the arguments below. First, we let $B_{1}, B_{2}, B_{3}, \cdots$ be defined as in the proof of Theorem 4.1, with $D \backslash \Gamma$ replaced by $\mathbb{R}^{3} \backslash \Gamma$. For $v \in \mathcal{S}$, and for any open ball $B \subset \mathbb{R}^{3} \backslash \Gamma$, we define $T_{B} v$, similar to the definition in the proof of of Theorem 4.1, only changing $e^{S}$ in (4.6) to 1. Define $\left\{v_{i}\right\} \subset \mathcal{S}$ the same as below (4.6). Since $v_{i} \leq \bar{u}$ in $M$, we deduce, using also the local convexity and the monotonicity property of $\left\{v_{i}\right\}$ (recall that $v_{i} \leq v_{i+1}$ in $M)$, that $v_{i}$ converges in $C_{l o c}^{0}(M)$ to some locally convex function $u$. Clearly $u$ satisfies (8.3) and (8.5). By some standard arguments, $u$ satisfies (8.2) in the viscosity sense. Since

$$
\limsup _{x \rightarrow \bar{x}}\left(v_{i}(x, g)-\underline{u}(x, g)\right) \leq 0 \quad \forall \bar{x} \in \Gamma, \forall g \in G,
$$

we can deduce (8.4) by using arguments similar to those used in the proof of Theorem 4.1. Theorem 8.1 is established.

\section{Appendix.}

Lemma 9.1. Let $D$ be a strictly convex bounded open set in $\mathbb{R}^{n}, n \geq 1$, with $C^{2}$ boundary, and let $\Phi \in C^{2}(\bar{D})$. Then, there exists some constant $C$, depending only on $n, \Phi$ and $D$, such that for every $\xi \in \partial D$, there exists $\bar{x}(\xi) \in \mathbb{R}^{n}$ satisfying

$$
|\bar{x}(\xi)| \leq C \text { and } w_{\xi}<\Phi \text { on } \bar{D} \backslash\{\xi\},
$$


where

$$
w_{\xi}(x):=\Phi(\xi)+\frac{1}{2}\left(|x-\bar{x}(\xi)|^{2}-|\xi-\bar{x}(\xi)|^{2}\right), \quad x \in \mathbb{R}^{n} .
$$

Proof. It follows from modification of the proof of lemma 5.1 in [6].

\section{References.}

[1] L. Caffarelli, Certain multiple valued harmonic functions, Proc. Amer. Math. Soc. 54 (1976), 90-92.

[2] L. Caffarelli, On the Hölder continuity of multiple valued harmonic functions, Indiana Univ. Math. J. 25 (1976), 79-84.

[3] L. Caffarelli, A localization property of viscosity solutions to the MongeAmpère equation and their strict convexity, Ann. of Math. 13 (1990), $129-134$.

[4] L. Caffarelli, Interior $W^{2, p}$ estimates for solutions of the MongeAmpère equation, Ann. Math. 131 (1990), 135-150.

[5] L. Caffarelli, A note on the degeneracy of convex solutions to MongeAmpère equations, Commun. in Partial Differential Equations 18 (1993), 1213-1217.

[6] L. Caffarelli and Y.Y. Li, An extension to a theorem of Jörgens, Calabi, and Pogorelov, Comm. Pure Appl. Math. 56 (2003), 549-583.

[7] L. Caffarelli, L. Nirenberg and J. Spruck, The Dirichlet problem for nonlinear second-order elliptic equations, I. Monge-Ampère equation, Comm. Pure Appl. Math. 37 (1984), 369-402.

[8] G.C. Evans, A necessary and sufficient condition of Wiener, Amer. Math. Monthly, 54 (1947), 151-155.

[9] G.C. Evans, Surfaces of minimal capacity, Proc. Nat. Acad. Sci. USA 26 (1940), 489-491.

[10] G.C. Evans, Lectures on multiple valued harmonic functions in space, Univ. Calif. Publ. Math. (N.S.) 1 (1951), 281-340. 
[11] O.D. Kellogg, Foundations of potential theory, New York, Dover, 1954.

[12] N.C. Leung, Geometric aspects of Mirror Symmetry (with SYZ for Rigid CY manifolds), arXiv:math.DG/0204168 v3 28 Mar 2003.

[13] H. Lewy, Generalization of a spatial angle theorem, translated from the English by Ju. V. Egorov, Uspehi Mat. Nauk 26 (1971), 2(158), 199-204.

[14] J. Loftin, S.T. Yau and E. Zaslow, Affine Manifolds, SYZ Geometry, and the "Y" Vertex, arXiv:math.DG/0405061 4 May 2004.

L. Caffarelli

Department of Mathematics

University of Texas, Austin

Austin, TX 78712

YanYan Li

Department of Mathematics

Rutgers University

110 Frelinghuysen Road

Piscataway, NJ 08854 